\title{
Preface
}

This issue contains refereed papers based on presentations at the Second International Conference on Modern Trends in Computational Physics held on July 24-29, 2000 in the Laboratory of Information Technologies, Joint Institute for Nuclear Research, Dubna, Russia. The conference follows the rich traditions of the previous conference Modern Trends in Computational Physics (1998) and others conferences on Numerical Methods and Applications that have been held in the LIT of JINR since 1964.

This conference is held in memory of professor N. N. Govorun.

\section{N.N.Govorun (1930-1989)}

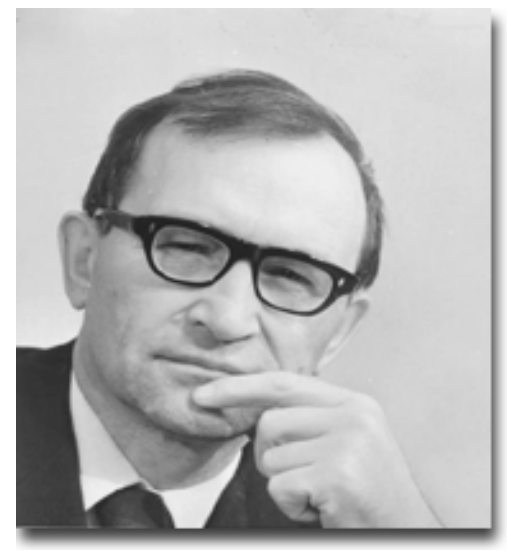

1953 - graduated from Moscow State University.

1969 - vice-director of the Laboratory of Computing Techniques and Automation, JINR.

1972 - selected a corresponding member of the USSR Academy of Sciences.

1988 - director of the Laboratory of Computing Techniques and Automation, JINR.

The most important stage of N.N.Govorun's activities was his work on the development of multi-computer complexes and corresponding system software of the physics research with wide areas of applications. The main result of the work was the creation of the advanced computing centre, the local computer network at the Joint Institute for Nuclear Research and first computer communication channels with other scientific centers.

The conference was organized by the Laboratory of Information Technologies (the former Laboratory of Computing Techniques and Automation) of the Joint Institute for Nuclear Research and co-organized by the Russian University of People's Friendship, under the sponsoring of the Ministry of Industry, Science and Technology, Russia, and Russian Foundation for Basic Research, Science Center for Applied Research, JINR.

About 130 scientists from 11 countries presented 19 plenary talks and 111 short communications at the conference. The objective was to provide a forum for the presentation of new approaches to computational modeling in physics, namely numerical and computer algebra methods and algorithms, advanced methods for boundary value problems, computer tools for simulation and experimental data analysis, computational tools for analysis of physical processes, complex systems 
modeling, mathematical modeling of nonlinear static and time-dependent physical fields, modeling exotic systems, Monte Carlo modeling of interactions of particles and of particles with matter, problems of nonlinear mathematical physics, modeling in ecology and biophysics, large-scale scientific computations on advanced computer architectures, and software for physics experiments.

We are grateful to all the authors for their contributions.

I.V. Puzynin 


\section{Acknowledgments}

I would like to express my thanks to all members of the International Advisory Board and Scientific Committee for their help in organizing the Conference. I appreciate the cooperation of MTCP'2000 Honorary Chairman, Professor E. P. Zhidkov, and LIT Deputy Directors, Drs. S. O. Lukianov and V. V. Korenkov. The hard work on this issue, as well as on technical and scientific organization of the Conference was shared by my colleagues, Drs. E. Ayrjan, A. Fedorov, B. Kostenko, I.Pokorný, O. Streltsova, S. Vinitsky and T. Strizh, and Mr. A. Lutsenko, Mrs. M. V. Aristarkhova. Without their efforts it would have been impossible to organize this Conference. I am also very grateful to Professors D. Morgan (Jr) and D. Truhlar for their collaboration and support of our conference proceedings in JCMSE. The success of the Conference was ensured in many respects by the efficiency of many offices of JINR. Special thanks are due to Mr. A. Korol from the International Department.

The financial support of the Russian Foundation for Basic Research, the Russian Ministry of Science and Technology, the Russian University of People's Friendship, and the Scientific Centre for Applied Research JINR is gratefully acknowledged.

I. V. Puzynin

MTCP'2000 Chairman 